\title{
A Rare Cause of Chronic Cough in Children: Lingual Thyroid
}

\author{
Weihua Wang, MD; Zhenghua Zhu, MD; Zhaoxin Ma, MD* \\ Department of Otolaryngology-Head \& Neck Surgery, Shanghai East Hospital, Tongji University School of Medicine, Shanghai, China
}

\begin{abstract}
Lingual thyroid is characterized by presence of thyroid tissue at the tongue base. Many patients are usually asymptomatic. In this report, we present a 14-year-old girl with chronic cough. The patient was referred to the ENT clinic with a history of fullness in her throat and irritative cough. Fiberolaryngoscope revealed a solid, spherical mass at the base of her tongue. Thyroid ultrasonography and scintigraphy showed a lingual thyroid. Lingual thyroid does not usually result in any symptoms unless an increase in gland size occurs. Endocrine changes can bring about an increase in gland size and symptoms. Our patient was managed conservatively, because there was no normal thyroid in the usual location of the neck. After 3 years of follow up, the mass was tolerated well and the patient became asymptomatic with a stable tissue volume.

Keywords: lingual thyroid; chronic cough; child
\end{abstract}

\section{Introduction}

The thyroid tissue reaches the normal location in the pretracheal region by migrating caudally via the foramen cecum in the base of the tongue at the seventh week of fetal life. Ectopic lingual thyroid is a developmental anomaly caused by noncompletion of this migration [1]. Lingual thyroid is characterized by presence of thyroid tissue at the tongue base in the region between the circumvallate papillae and the epiglottis, which is the most common location for functional ectopic thyroid tissue [2]. The pathogenesis of this occurrence is currently not known. The prevalence of lingual thyroid varies from 1:100,000 to $1: 300,000$ and may remain undiagnosed for a long period in life [3]. It is reported as being 4-8 times more common in females [4]. The average age at presentation was 40.5 years with statistically significant peak at 12.5 and 50 years.

Many patients are usually asymptomatic, but may show symptoms in some cases $[1,5]$. The most common symptoms found in adults include respiratory problems, swallowing difficulties, foreign body sensation in the throat, hemorrhage and dysphonia $[3,6]$. Symptomatic lingual thyroids more often emerge during puberty, pregnancy, menstruation or any other stressful event [7]. Thus, the clinical presentation of lingual thyroid in pediatric patients is innumerable. In this report, we present a 14-yearold girl with lingual thyroid resulting in chronic irritative cough and a review of the relevant literature.

\section{Case Report}

A 14-year-old girl presented to our clinic with a history of fullness in her throat and irritative cough for the last 5 months. No respiratory problems, swallowing difficulties or dysphonia are reported. Clinical examination using fiberolaryngoscope revealed a solid, spherical mass formed at the base of her tongue, filling the epiglottic vallecula (Figure 1). She had no past or present history of thyroid disease. Examination of the neck revealed no palpable thyroid gland in the pretracheal position and also no cervical adenopathy. Ultrasonography (USG) of the neck revealed no echogenicity indicative of thyroid gland in its original anatomical location.

*Correspondence: Zhaoxin Ma, MD

Department of Otolaryngology-Head \& Neck Surgery, Shanghai East Hospital, Tongji University School of Medicine, Shanghai, China. E-mail: whwangcn@tongji.edu.cn

Received: April 22, 2017; Accepted: May 12, 2017; Published: May 16, 2017

Archives of Otorhinolaryngology-Head \& Neck Surgery. 2017;1(1):4 DOI: 10.24983/scitemed.aohns.2017.00012

Copyright (c) 2017 The Author(s). This is an open-access article distributed under the terms of the Creative Commons Attribution 4.0 International License (CC-BY).
However, there was an echogenicity of a solid mass formation at the base of the tongue. The mass was $2.5 \mathrm{~cm}$ in diameter and had well-defined contours. Thyroid scintigraphy showed an uptake region at the base of the tongue, signifying a lingual thyroid. There was no thyroid uptake in the usual location of the neck (Figure 2). Thyroid hormone tests revealed normal serum levels of thyroid stimulating hormone, free T3, and free T4. She had no history of asthma, allergic rhinitis or postnasal drip. The patient was informed that the onset of cough was related to periods of high metabolic activity, such as puberty. Conservative management with close monitoring was recommended. No thyroid hormone replacement was initiated. After 3 years of follow up, the mass was tolerated well and the patient was asymptomatic with a stable tissue volume.

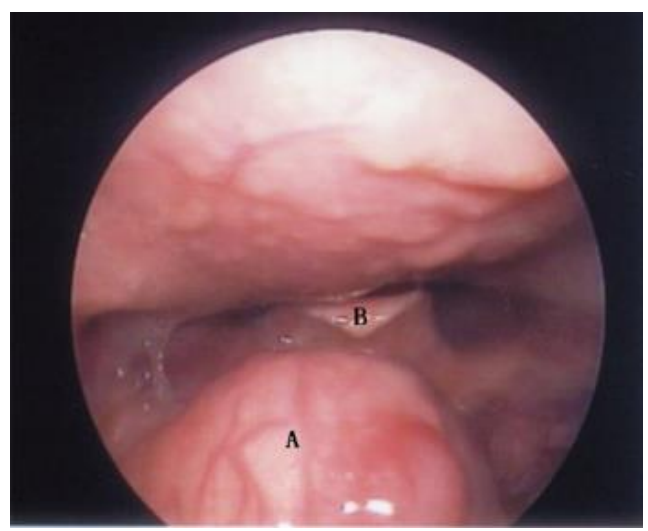

Figure 1. Endoscopic image of lingual thyroid. A, a hemispheric and firm mass attached to the tongue base in the region of the foramen caecum; B, epiglottis.

\section{Discussion}

Thyroid gland ectopia has been reported in the oropharynx, infrahyoid region, larynx, mediastinum, esophagus, porta hepatis and heart $[6,8]$. The most common location of aberrant thyroid tissue is the tongue, as $90 \%$ of ectopic glands are identified at the tongue base. Lingual thyroid is a rare developmental anomaly that occurs subsequent to the failed caudal migration of the thyroid gland from the ventral pharynx to the normal pretracheal position in the neck. Almost one third of patients have overt hypothyroidism at the time of diagnosis [9]. When examined histopathologically, it is observed that the lingual thyroid tissue contains benign thyroid gland tissue, in most cases. However, carcinoma within a lin- 
gual thyroid gland has been reported [9]. Early detection and appropriate treatment of lingual thyroid are crucial for optimal neurological development and physical growth, especially in children [10]. In this report, we discuss the case report of a 14-year-old girl with lingual thyroid, which was fortunately detected at an early stage.

Usually, lingual thyroid does not produce any symptoms unless an increase in gland size occurs. Frequently experienced symptoms in these patients are due to mass effect, dysphonia, dysphagia, lump in throat sensation and dyspnea [9]. Endocrine changes such as puberty, pregnancy and menstruation can lead to an increase in gland size and symptoms. This explains why lingual thyroid occurs largely among women. Our young patient reported fullness in her throat and chronic irritative cough in her puberty. Hence, lingual thyroid should be suspected if chronic cough prevails, especially in young female patients.

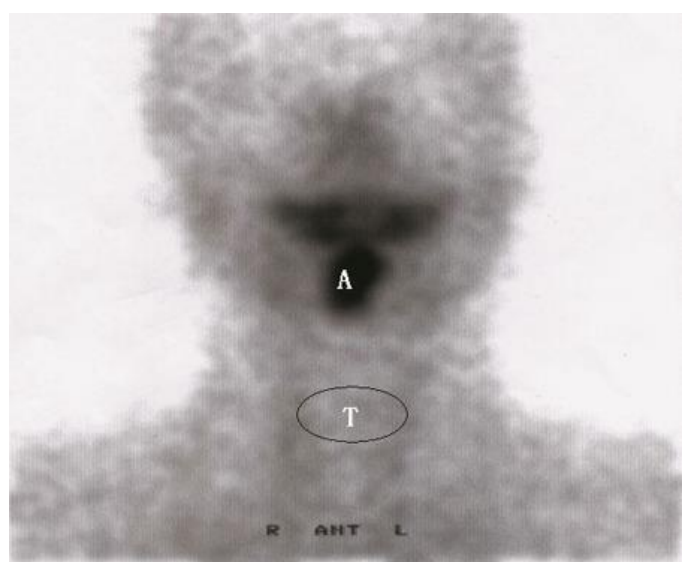

Figure 2. Thyroid scintigraphy with technetium (Tc99m), revealing isotope uptake at the base of the tongue (A); and no uptake in the normal thyroid location (T).

The normal thyroid gland, which can be seen together with ectopic thyroid tissues, needs to be explored. A normally located thyroid is seen in $30 \%$ of patients with lingual thyroid. In those patients in whom the thyroid gland is not seen in the normal location, the lingual thyroid must be detected. USG is one of the simplest methods for thyroid scan, in which lingual thyroid is seen with regular contours and homogenously with more echogenicity compared to tongue muscles. In our case, there was no echogenicity in the normal location. Thyroid scintigraphy is suggested for the differential diagnosis of tongue base masses detected on physical examination or when thyroid tissue is not detected in the normal location on USG. In the scintigraphic evaluation, activity similar to normally located thyroid is detected in ectopic thyroid tissue. In our case, while the activity was not detected in the normal location, thyroid activity was detected at the tongue base. Diseases such as adenoma, hyperplasia, inflammation and carcinoma, which affect the normal thyroid, can also be seen in ectopic thyroid gland. In the differential diagnosis, fibromas, lipomas, hemangiomas, granular cell myoblastomas, thyroglossal duct cysts, minor salivary gland tumors and squamous cell carcinomas that can be seen in this region must also be taken into consideration. Biopsy is not recommended because of the risks of bleeding and infection [1].

Lingual thyroid could be the only functional thyroid tissue. Asymptomatic cases can be monitored with suppressive hormone. In some of the cases, suppressive treatment can decrease the tissue volume. Surgical indications for lingual thyroid include dyspnea, dysphagia and suspicion of malignancy, uncontrolled hyperthyroidism, and repetitive bleedings of serious nature. Surgical excision can be made either transorally or externally with median lateral pharyngotomy following a transhyoidal approach. Extreme caution must be taken while selecting the method of treatment, especially, for the children during puberty. In such situations, clinicians are indeed caught in a dilemma between the symptomatic mass and maintaining thyroid function. Therefore, close monitoring will be the best option. Otherwise, suppressive therapy should be tried first, which may help to reduce the size of the gland or at least keep it from enlarging. If the patient does not respond well, surgery may have to be considered. Thyroid function should be detected during the treatment. In our case, the girl could well tolerate the lingual thyroid. One possible reason is that the oropharyngeal cavity becomes bigger gradually with ages and the relative size of the lingual thyroid decreases, thus reducing the irritancy of ectopic thyroid.

\section{Conclusion}

Clinicians should remain on high alert for oropharyngeal masses while evaluating chronic cough patients. When a mass lesion is observed in the tongue base, ectopic lingual thyroid must be considered in the differential diagnosis, and the diagnosis must be verified using USG and scintigraphy. It is important to pay more attention to a local irritation that is related to the increased physiological demand for thyroxine during puberty and pregnancy.

References

1. Toso A, Colombani F, Averono G, Aluffi P, Pia F. Lingual thyroid causing dysphagia and dyspnoea. Case reports and review of the literature. Acta Otorhinolaryngol Ital 2009; 29:213-217.

2. Ulug T, Ulubil SA, Alagol F. Dual ectopic thyroid: report of a case. J Laryngol Otol 2003; 117:574-576.

3. Wu ZX, Zheng LW, Dong YJ, et al. Modified approach for lingual thyroid transposition: report of two cases. Thyroid 2008; 18:465-468.

4. Peters P, Stark P, Essig GJ, et al. Lingual thyroid: an unusual and surgically curable cause of sleep apnoea in a male. Sleep Breath 2010; 14: 377-380.

5. Barnes TW, Olsen KD, Morgenthaler TI. Obstructive lingual thyroid causing sleep apnea: a case report and review of the literature. Sleep Med 2004; 5:605-607.

6. Rahbar R, Yoon MJ, Connolly LP, et al. Lingual thyroid in children: a rare clinical entity. Laryngoscope 2008; 118: 1174-1179.

7. Chiu TT, Su CY, Hwang CF, Chien CY, Eng HL. Massive bleeding from an ectopic lingual thyroid follicular adenoma during pregnancy. Am J Otolaryngol 2002; 23:185-188.

8. Ghanem N, Bley T, Altehoefer C, Högerle S, Langer M. Ectopic thyroid gland in the porta hepatis and lingua. Thyroid 2003; 13:503-507.

9. Oppenheimer R. Lingual thyroid associated with chronic cough. Otolaryngol Head Neck Surg 2001; 125:433-434.

10. Oerbeck B, Sundet K, Kase BF, Heyerdahl S. Congenital hypothyroidism: influence of disease severity and L-thyroxine treatment on intellectual, motor, and school-associated outcomes in young adults. Pediatrics 2003; 112:923-930. 